\title{
FUNCIONALIDAD FAMILIAR Y DETERIORO COGNITIVO EN LOS ADULTOS MAYORES DE LA ZONA 7. ECUADOR, 2016
}

\author{
FAMILY FUNCTIONALITY AND COGNITIVE IMPAIRMENT \\ IN OLDER ADULTS IN ZONE 7. ECUADOR, 2016
}

FUNCIONALIDADE FAMILIAR E DETERIORAÇÃO COGNITIVA EM ADULTOS IDOSOS DA ZONA 7. EQUADOR, 2016

\author{
JAIME OSWALDO TIGRE BUENO', CLEYNE RODRÍGUEZ PARDILLO', JOSÉ GONZÁLEZ ESTRELLA', CRISTINA ALDAZ \\ BARRENA'
}

Universidad de Loja, Loja-Ecuador

RESUMEN

Objetivo: determinar la relación entre la funcionalidad familiar y el deterioro cognitivo de los adultos mayores de la Zona 7 del Ecuador. Materiales y métodos: estudio descriptivo, transversal, realizado en mayo a julio de 2016 en las provincias de Loja, EL Oro y Zamora Chinchipe pertenecientes a la Zona de Planificación 7. Se recolectaron datos sociodemográficos de 1.146 adultos mayores de la región sur del Ecuador. Se valoró la funcionalidad familiar mediante el test de funcionamiento familiar FF-SIL. El estado cognitivo fue evaluado mediante el cuestionario de Actividad Funcional de Pfeffer (PFAQ). Resultados: la prevalencia de disfuncionalidad familiar fue de 30.8\% y de disfuncionalidad severa de $14,6 \%$. La prevalencia de deterioro cognitivo en los adultos mayores fue $25,5 \%$. Se encontró mayor prevalencia de deterioro cognitivo en los ancianos de familias disfuncionales que en los adultos mayores de familias funcionales encontrándose relación estadísticamente significativa ( $p<0,001)$. Conclusión: se encontró asociación estadística entre la funcionalidad familiar y el deterioro cognitivo en los ancianos. Los adultos mayores con deterioro cognitivo requieren una aproximación holística e integral que abarque al sistema familiar para indagar problemas que pueden intervenir en el proceso salud enfermedad.

PALABRAS CLAVE: familia, anciano, FF-SIL, cognición, disfunción familiar.

ABSTRACT

Objective: determine the relationship between family functionality and cognitive impairment in older adults in Zone 7 of Ecuador. Materials and methods: descriptive, cross-sectional study, carried out in May and July 2016 in the provinces of Loja, El Oro, and Zamora Chinchipe belonging to the Planning Zone 7. Sociodemographic data collected from 1,146 older adults in the southern region of Ecuador. Family functionality assessed using the FF-SIL family functioning test and Cognitive status assessed using the Pfeffer, Functional Activities Questionnaire (FAQ). Results: the prevalence of family dysfunction was $30.8 \%$ and severe dysfunction of $14.6 \%$. The prevalence of cognitive impairment in older adults was $25.5 \%$. A higher prevalence of cognitive impairment was found in the elderly from dysfunctional families than in the elderly from functional families, finding a statistically significant relationship $(\mathrm{p}<0.001)$. Conclusion: a statistical association found between family functionality and cognitive impairment in the elderly. Older adults with cognitive impairment require a holistic and comprehensive approach that encompasses the family system to investigate problems that may intervene in the health-disease process.

KEYWORDS: family, aged, FF-SIL, cognition, family dysfuntion.

RESUMO

Objetivo: determinar a relação entre funcionalidade familiar e prejuízo cognitivo em idosos da Zona 7 do Equador. Materiais e métodos: estudo descritivo transversal, realizado em maio e julho de 2016 nas províncias de Loja, EL Oro e Zamora Chinchipe pertencentes à Zona de Planejamento 7. Os dados sociodemográficos foram coletados de 1.146 idosos na região sul do Equador. A funcionalidade familiar foi avaliada usando 0 teste de funcionamento familiar FF-SIL. 0 estado cognitivo foi avaliado através do questionário Pfeffer Functional Activity (PFAQ). Resultados: a prevalência de disfunção familiar foi de 30,8\% e de disfunção grave de 14,6\%. A prevalência de déficit cognitivo em idosos foi de 25,5\%. Maior prevalência de déficit cognitivo foi encontrada em idosos de famílias disfuncionais do que em idosos de famílias funcionais, encontrando relação estatisticamente significativa $(p<0,001)$. Conclusão: foi encontrada associação estatística entre funcionalidade familiar e déficit cognitivo em idosos. Os idosos com deficiência cognitiva requerem uma abordagem holística e abrangente que englobe 0 sistema familiar para investigar os problemas que podem intervir no processo saúde-doença.

PALAVRAS-CHAVE: família, idoso, FF-SIL, cognição, disfunção familiar. 
INTRODUCCIÓN

Según la teoría general de sistemas, la familia es un microsistema que en el curso del tiempo ha elaborado pautas de interacción que rigen el funcionamiento de los miembros de la familia, definen conductas y facilitan sus interacciones recíprocas. ${ }^{1}$ La familia tienen dos objetivos: la protección psicosocial de sus miembros y la transmisión de una cultura. ${ }^{2}$ En el mundo occidental el grupo nuclear característico es el conformado por el padre, la madre y los hijos. ${ }^{3}$ La familia latinoamericana ha sufrido cambios, durante los últimos 25 años, con el aumento de las familias en fase de contracción, ancianos viviendo solos y familias sin hijos. ${ }^{4}$

El cambio de estos modelos familiares se relaciona con el aumento de la población anciana. En el año 1950 el porcentaje mundial de ancianos alcanzaba el $8 \%$ mientras que para el año 2050 , esta prevalencia se triplicará; globalmente la población de adultos mayores crece a una tasa de $2.6 \%$ por año, mucho más que la población general. ${ }^{5}$ En Ecuador la población de adultos mayores es de 1'229.089 individuos mayores de 60 años. En la Zona de Planificación 7 hay 87.685 personas adultos mayores que representan el 7,3 por ciento de la población. ${ }^{6}$

Este envejecimiento poblacional resulta en el aumento de la prevalencia de enfermedades crónicas-degenerativas, como las alteraciones de la cognición. La función cognitiva es la facultad de procesar la información a partir de la percepción y la experiencia. El funcionamiento cognitivo se diferencia en varios dominios: aprendizaje, memoria, lenguaje, visuoespacial, ejecutivo y psicomotor. ${ }^{7}$ La alteración de la función cognitiva, que comprende desde el deterioro cognitivo leve hasta la demencia grave, puede interferir con las actividades de la vida diaria del anciano.

En Ecuador el deterioro cognitivo se presenta en el $21,3 \%$ de los adultos mayores de 60 años; ${ }^{8}$ La importancia del diagnóstico del deterioro cognitivo radica en la intervención oportuna de sus factores de riesgo y la adecuada evaluación del funcionamiento familiar por cuanto varias investigaciones encuentran asociación entre la funcionalidad familiar y el deterioro cognitivo de los ancianos. ${ }^{9}$ Un estudio cubano señala la presencia de disfuncionalidad familiar en 52,5\% de los ancianos con deterioro cognitivo. ${ }^{10}$

En el ámbito de la medicina familiar en Ecuador existen pocas investigaciones relacionadas con la dinámica familiar y su participación en el proceso salud-enfermedad. Por tanto el objetivo principal de esta investigación es determinar la funcionalidad familiar y su relación con el estado cognitivo del anciano, con la hipótesis que existe asociación estadística entre la funcionalidad familiar y el deterioro cognitivo de los adultos mayores.

\section{METODOLOGÍA}

La presente investigación descriptiva, y transversal se desarrolló en la Zona 7, ubicada al sur del Ecuador, conformada por las provincias de El Oro, Loja y Zamora Chinchipe, como parte del Macroproyecto "Calidad de vida del Adulto Mayor de la Zona 7". El universo fue conformado por la población de adultos mayores según la proyección del Instituto Ecuatoriano de Estadística y Censo (INEC) 2016, corresponde a 99563 adultos mayores como población objetivo. Se trata de una muestra probabilística por racimos determinada informáticamente por el programa EPI INFOTM 7.1.4.0 condicionado por una frecuencia esperada del $50 \%$, límite de confianza del $5 \%$, nivel de confianza del 95\%, defecto de diseño del 0,1 y 1 clúster por cada provincia, donde las unidades de análisis son los adultos de 65 y más años, para El Oro: 385, Loja: 395 y Zamora Chinchipe: 366, para un total de 1146 unidades de análisis, con una potencia estadística del $85 \%$ verificado por el software $G^{*}$ Power Versión 3.0.10. El muestreo fue aleatorio estratificado dividiendo a la población en estratos por parroquias, piso altitudinal, que tienen alguna característica común, utilizando hojas de cálculo previamente diseñadas utilizando Microsoft Office Excel ${ }^{\circledR} 2010$ (Microsoft Corporation, EEUU).

El control de la calidad de la información a recolectar se efectuó mediante una prueba piloto, en 30 sujetos de similares características a la población estudiada, sin que ésta forme parte de la investigación. La recolección de datos de los usuarios se realizó durante los meses de mayo y julio de 2016 en las tres provincias descritas. Los criterios de inclusión para la población seleccionada fueron: edad de 65 años o más. Se excluyó a los ancianos que no deseaban colaborar en el estudio o cuyo cuidador no deseaba consentir la participación. Se firmó el consentimiento informado por el adulto mayor o el informante de acuerdo a las normas de Helsinki.

La información se recolectó mediante tres instrumentos: 
1. Datos sociodemográficos del adulto mayor.

2. Se utilizó un test elaborado en Cuba, la prueba de Percepción del Funcionamiento Familiar (FF-SIL), dirigido al adulto mayor o informante para la valoración de la funcionalidad familiar; consta de 14 ítems y el puntaje final clasifica la familia en funcional, moderadamente funcional, disfuncional y severamente disfuncional. ${ }^{11}$

3. Valoración de la situación cognitiva del anciano aplicada al informante o cuidador mediante la Escala de Actividades Funcionales de Pfeffer (PFAQ, por sus siglas en inglés), ${ }^{12}$ instrumento que consta en los protocolos del Ministerio de Salud Pública del Ecuador; ${ }^{33}$ presenta 11 ítems y una puntuación de 6 o más define deterioro cognitivo con una adecuada sensibilidad y especificidad. ${ }^{14}$ Para verificar las asociaciones entre las variables situación cognitiva y funcionalidad familiar fueron evaluadas mediante pruebas de chi cuadrado. Las variables que se estudiaron fueron nivel de función cognitiva (cognición normal o deterioro cognitivo) y funcionalidad familiar (funcional o disfuncional).La información compilada se analizó en el software SPSS ${ }^{\circledR}$ versión 16 (IBM Corporation, EEUU), aplicando el método porcentual según el caso y la prueba no paramétrica del Chi-cuadrado para determinar asociación.

RESULTADOS

Las características socio-demográficas de los 1146 ancianos incluidos en esta investigación se resumen en la tabla 1. El grupo de los ancianos jóvenes (65 - 74 años) representa la mitad de la población estudiada. Los mayores de 80 años, denominado la "cuarta edad" o los "viejos-viejos", representa cerca de la tercera parte de la población estudiada, mayor a la cifra nacional. ${ }^{6}$

TABLA 1. CARACTERIZACIÓN DE 1146 ADULTOS MAYORES DE LAZONA 7

\begin{tabular}{llrr} 
VARIABLE & & NÚMERO & PORCENTAJE \\
\hline \multirow{2}{*}{ EDAD } & 65 a 74 años & 603 & 52,7 \\
& 75 a 79 años & 216 & 18,8 \\
& 80 años y más & 327 & 28,5 \\
SEXO & Mujer & 556 & 48,5 \\
& Hombre & 590 & 51,5 \\
ÁREA DE & Urbano & 706 & 61,6 \\
PROCEDENCIA & Rural & 440 & 38,4 \\
\multirow{2}{*}{ FUNCIONALIDAD } & Severamente disfuncional & 167 & 14,6 \\
FAMILIAR & Disfuncional & 353 & 30,8 \\
& Moderadamente funcional & 396 & 34,6 \\
& Funcional & 230 & 20,1
\end{tabular}

Fuente: base de datos: macroproyecto: calidad de vida del adulto mayor de la zona 7,2016 .
Los ancianos de la cuarta edad se considera una población sobreviviente que ha superado la expectativa de vida y cuenta con la carga más alta de enfermedad degenerativa y discapacidad. ${ }^{15}$

Existen similares porcentajes en la población estudiada según el sexo, y la mayoría de los ancianos son de procedencia urbana. Según el FF-SIL el 45,4\% de los adultos mayores viven en familias disfuncionales; $14,6 \%$ de adultos mayores están expuestos a sistemas familiares con severa disfunción.

El 25,5\% de los adultos mayores de 65 años presentan deterioro cognitivo (Tabla № 2). Existe una mayor prevalencia en ancianos de la cuarta edad $(45,57 \%)$ comparado a los grupos de ancianos jóvenes con una asociación estadística significativa. No existen diferencias significativas en cuanto el sexo o el área de procedencia del anciano. Se reveló una prevalencia alta de disfuncionalidad familiar $(29,8 \%)$ y severa disfunción (25\%) entre los ancianos con deterioro cognitivo. La relación entre la disfuncionalidad familiar y el deterioro cognitivo de los adultos mayores es significativa con una $\mathrm{p}<0,05(\mathrm{p}<0,001)$.

TABLA 2. DISTRIBUCIÓN DE 1.146 ADULTOS MAYORES DE LA ZONA 7 SEGÚN SITUACIÓN COGNITIVA.

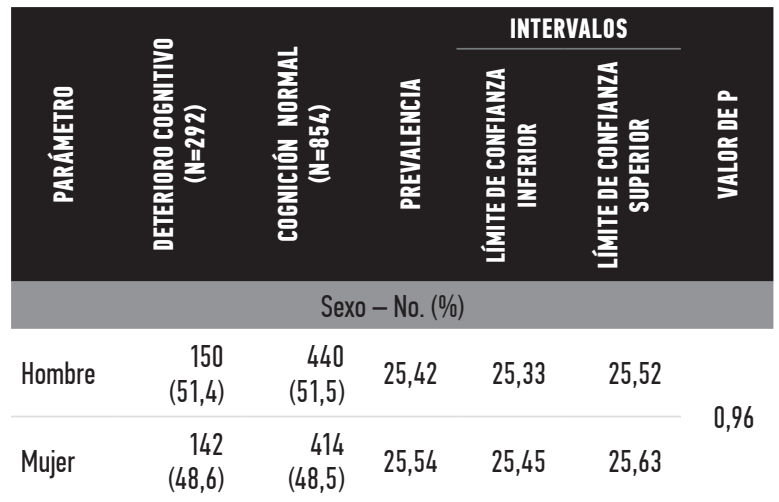

Edad - No. (\%)

\begin{tabular}{|c|c|c|c|c|c|c|}
\hline $\begin{array}{l}65 \text { a } 74 \\
\text { años }\end{array}$ & $91(31,2)$ & $512(60)$ & 15,09 & 13,30 & 16,89 & \\
\hline $\begin{array}{l}75 \text { a } 79 \\
\text { años }\end{array}$ & $52(17,8)$ & $\begin{array}{r}164 \\
(19,2)\end{array}$ & 24,07 & 22,28 & 25,87 & $\begin{array}{r}< \\
0,001\end{array}$ \\
\hline $\begin{array}{l}80 \text { años y } \\
\text { más }\end{array}$ & $149(51)$ & $\begin{array}{r}178 \\
(20,8)\end{array}$ & 45,57 & 43,77 & 47,36 & \\
\hline \multicolumn{7}{|c|}{ Área de procedencia - №. (\%) } \\
\hline Urbana & $\begin{array}{r}185 \\
(63,4)\end{array}$ & $521(61)$ & 26,20 & 26,05 & 26,36 & \multirow[b]{2}{*}{0,47} \\
\hline Rural & $\begin{array}{r}107 \\
(36,6)\end{array}$ & 333 (39) & 24,32 & 24,17 & 24,47 & \\
\hline
\end{tabular}

Fuente: base de datos: macroproyecto: calidad de vida del adulto mayor de la zona 7,2016. 
TABLA N 3. DISTRIBUCIÓN DE 1146 ADULTOS MAYORES DE LA ZONA 7 SEGÚN FUNCIONALIDAD FAMILIAR

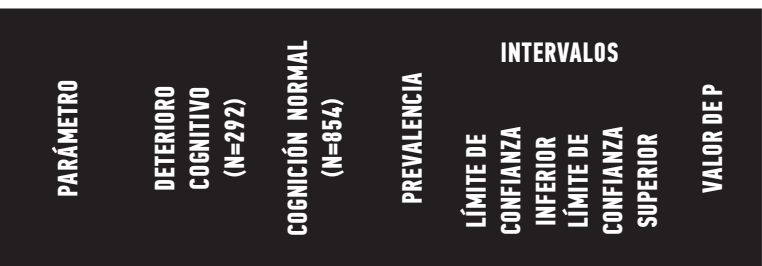

Funcionalidad familiar - №. (\%)

\begin{tabular}{|c|c|c|c|c|c|}
\hline $\begin{array}{l}\text { Severa- } \\
\text { mente } \\
\text { disfuncio- } \\
\text { nal }\end{array}$ & $73(25)$ & 94 (11) & 43,71 & 43,56 & 43,87 \\
\hline $\begin{array}{l}\text { Disfun- } \\
\text { cional }\end{array}$ & $87(29,8)$ & $\begin{array}{r}266 \\
(31,1)\end{array}$ & 24,65 & 24,49 & 24,80 \\
\hline $\begin{array}{l}\text { Modera- } \\
\text { damente } \\
\text { funcional }\end{array}$ & $74(25,3)$ & $\begin{array}{r}322 \\
(37,7)\end{array}$ & 18,69 & 18,53 & 18,84 \\
\hline Funcional & $58(19,9)$ & $\begin{array}{r}172 \\
(20,1)\end{array}$ & 25,22 & 25,06 & 25,37 \\
\hline
\end{tabular}

Fuente: base de datos: macroproyecto: calidad de vida del adulto mayor de la zona, 7.2016.

DISCUSIÓN

La prevalencia de deterioro cognitivo en la Zona Sur $(25,5 \%)$ es mayor a la encontrada a nivel nacional; según la Encuesta de Salud, Bienestar y Envejecimiento, que utilizó el instrumento FAQ para valoración de la cognición, y que encontró el 21,3\% de disfunción cognitiva en los adultos mayores de 60 años. ${ }^{1}$ Esta misma investigación encontró prevalencias bajas en zonas urbanas de Quito (16,2\%) y Guayaquil (17,6\%), mientras que prevalencias elevadas se hallaron en el sector rural de la costa $(22,6 \%$ y la región sierra $(36,1 \%)$; cifras aún más bajas si se comparan con estudios internacionales. ${ }^{16}$

En esta investigación se encontraron cifras altas de disfuncionalidad familiar $(54,4 \%)$. En una población general institucionalizada con trastornos mentales en México halló el 33\% de disfuncionalidad. ${ }^{17}$ Una publicación española planteó una prevalencia del $11 \%$ de disfuncionalidad grave a nivel de la población general; ${ }^{18}$ el 32,7\% de disfuncionalidad se encontró en una exploración brasileña entre ancianos mayores de 80 años. ${ }^{19}$ Cabe anotar que los artículos citados, así como la mayoría de investigaciones internacionales, el instrumento más usado para la valoración de la funcionalidad es el Apgar familiar como en una experiencia investigativa realizada en China donde más de la mitad de los ancianos de la cuarta edad tenían disfunción familiar $(52,2 \%)$, pero solamente el $8,6 \%$ mostraba disfunción familiar severa. ${ }^{9}$ Un estudio cubano encontró el $32,3 \%$ de disfunción familiar entre los adultos mayores utilizando el FF-SIL. ${ }^{20}$
La familia es un sistema compuesto por individuos que interactúan y modifica al resto de miembros y en consecuencia a toda la familia. ${ }^{21}$ Cuando un sistema familiar atraviesa por diversas crisis (normativas o paranormativas) puede perder su funcionalidad y expresarse en un miembro de la familia a través de la enfermedad. Esto a su vez desencadena o agrava un inadecuado funcionamiento familiar. ${ }^{22}$ El hallazgo de un anciano con deterioro cognitivo precisa la búsqueda de problemas que alteren al sistema familiar. Desde el diagnóstico de deterioro cognitivo, el seguimiento es crucial; la dinámica familiar cambia en dos aspectos: de manera centrípeta, al enfocar la atención de un anciano previamente desatendido; o de forma centrífuga, con el abandono de los parientes al ser la causa de una crisis paranormativa por desorganización. ${ }^{23}$

La progresión del deterioro cognitivo en el anciano significa inactividad laboral, social y la esperanza de un futuro independiente. Se redistribuyen las tareas y roles desempeñados por cada miembro familiar tanto en las situaciones estables como en las recaídas. La mayoría de las veces la responsabilidad y los cuidados del enfermo es delegada a una sola persona quien a largo plazo sufre exigencias por el resto de la familia ${ }^{24}$ hasta llegar a la disfunción, desintegración de los miembros de la familia y al colapso del cuidador. ${ }^{25}$

\section{CONCLUSIONES Y RECOMENDACIONES}

Se encontró asociación estadística entre la funcionalidad familiar y el deterioro cognitivo en los ancianos de la Zona 7. Los adultos mayores con deterioro cognitivo requieren una aproximación holística e integral que abarque al sistema familiar para indagar problemas que pueden intervenir en el proceso salud-enfermedad. La calidad de vida del anciano y sus familiares, dependerá del apoyo recibido del sistema de salud a través del médico familiar.

\section{REFERENCIAS BIBLIOGRÁFICAS}

1. Minuchin S, Fishman C. Técnicas de terapia familiar. 1a ed. Buenos Aires: Paidós; 2004. 288 p.

2. Minuchin S. Familias y terapia familiar. México: Ed. Gedisa; 2003. 349 p.

3. Huerta JL. Medicina familiar. La familia en el proceso salud-enfermedad. Aldrete J, editor. México: Ed.Alfil; 2005. 203 p.

4. Ullmann H, Valera CM, Rico MN. La evolución de las estructuras familiares en América Latina, 1990-2010; los retos de la pobreza, la vulnerabili- 
dad y el cuidado [Internet]. 2014. Available from: https://www.unicef.org/lac/S2014182_es.pdf

5. United Nations. World Population Ageing 2009. New York; 2009.

6. INEC. Censo de Población y Vivienda - Ecuador. 2010 [Internet]. 2010. 2015. Available from: http:// www.ecuadorencifras.gob.ec/censo-de-poblacion$\mathrm{y}^{-}$vivienda/

7. Gerrig F, Richard J, Psychology PGZ, Copyright MA, Education P. Glossary of Psychological Terms [Internet]. 2015 [cited 2015 Dec 18]. p. 12-4. Available from: http://www.apa.org/research/action/ glossary.aspx?tab=3

8. Freire WB. Primera Encuesta de Salud, Bienestar y Envejecimiento en Ecuador en adultos mayores (SABE I). Ecuador; 2010.

9. Wang B, He P, Dong B. Association between family functioning and cognitive impairment among Chinese nonagenarians / centenarians. Geriatr Gerontol Int [Internet]. 15(9):1135-42. Available from: http://onlinelibrary.wiley.com/doi/10.1111/ ggi.12410/epdf

10. Avila M. Caracterización de la familia del anciano con deterioro cognitivo. Rev Cub Salud Publica. 2012;38(2):246-52.

11. Ortega T. Propuesta de un instrumento para la aplicación del proceso de atención de enfermería en familias disfuncionales. Rev Cuba Enfermer [Internet]. 1999;15(3):164-8. Available from: http://www.bvs.sld.cu/revistas/enf/vol15_3_99/ enf05399.pdf

12. Pfeffer RI, Kurosaki TT, Harrah $\mathrm{CH}$, Chance JM, Filos S. Measurement of Functional Activities in Older Adults in the Community. J Gerontol. 1982;37(3):323-9.

13. Álvarez P, Martínez D. Guías clínicas gerontogeriátricas de Atención Primaria de Salud para el adulto mayor. Quito: MInisterio de Salud Pública del Ecuador; 2008. p. 186.

14. Quiroga P, Albala C, Klaasen G. Validación de un test de tamizaje para el diagnóstico de demencia asociada a edad, en Chile. Rev Med chile [Internet]. 2004;1(132):467-78. Available from: http:// www.scielo.cl/pdf/rmc/v132n4/art09.pdf

15. Valdivia G, Dominguez A. Población de 80 y más años en Chile: Una visión preliminar desde el punto de vista epidemiológico. REV MED CLIN CONDES. 2012;23(1):5-12.
16. Diaz R, Marulanda F, Martínez M. Prevalencia de deterioro cognitivo y demencia en mayores de 65 años en una población urbana colombiana. Acta Neurol Colomb. 2013;29(3):141-51.

17. Casanova-rodas L, Rascón-gasca ML, Alcántarachabelas H, Soriano-rodríguez A. Apoyo social y funcionalidad familiar en personas con trastorno mental. Salud Ment. 2014;37(5):443-8.

18. Revilla L de la. Factores que intervienen en la producción de los problemas psicosociales. Atención Primaria. 2007;39(6):305-11.

19. Porto D, Hisako L, Gonçalves T, Arminda M, Costa M, Martins MM, et al. La dinámica de la familia de ancianos con edad avanzada en el contexto de la ciudad de Porto, Portugal. Rev Latino-Am Enferm [Internet]. 2011;19(3):9. Available from: http:// www.scielo.br/pdf/rlae/v19n3/es_03.pdf

20. María M, Martín P, Cruz AD, Naranjo JA, González KÁ, Caridad R, et al. Medio familiar de los adultos mayores. Rev Ciencias Médicas. 2012;16(1):97-104.

21. Herrera P. La familia funcional y disfuncional , un indicador de salud. Rev Cuba Med Gen Integr. 1997;13(6):5-8.

22. Ortíz M. La salud familiar. Rev Cuba Med Gen Integr [Internet]. 1999;15(4):439-45. Available from: http://bvs.sld.cu/revistas/mgi/vol15_4_99/ mgi17499.pdf

23. Herrera P. Factores de riesgo para la salud familiar: acontecimientos significativos. Humanidades Médicas [Internet]. 2012;12(2):184-91. Available from: http://scielo.sld.cu/pdf/hmc/v12n2/hmc03212.pdf

24. Castillo IA. La carga de los cuidadores de adultos mayores con demencia en una región rural. Psicol y Salud [Internet]. 2014;24(55):35-43. Available from: http://revistas.uv.mx/index.php/psicysalud/ article/download/718/1265

25. Mateo Rodríguez I, Millán Carrasco a., García Calvente MM, Gutiérrez Cuadra P, Gonzalo Jiménez E, López Fernández L a. Cuidadores familiares de personas con enfermedad neurodegenerativa: perfil, aportaciones e impacto de cuidar. Atención Primaria [Internet]. Elsevier; 2000;26(3):139-44. Available from: http://linkinghub.elsevier.com/ retrieve/pii/S0212656700786306 